\title{
Paclitaxel-carboplatin chemotherapy induced hematologic toxicities among epithelial ovarian cancer patients
}

\author{
Afandi Charles*, Birgitta M. Dewayani**, Edhyana Sahiratmadja***, \\ Gatot N.A. Winarno****, and Herman Susanto****
}

\begin{abstract}
BACKGROUND
Epithelial ovarian cancer (EOC) is one of the most common cancers diagnosed in Indonesian women. A combination of paclitaxel and carboplatin is used to treat EOC as standard chemotherapy which is known to have hematologic toxicities. This study aimed to investigate the effect of combined paclitaxel-carboplatin chemotherapy on hematologic status in EOC patients managed at Dr. Hasan Sadikin General Hospital, Bandung, West Java.
\end{abstract}

\section{METHODS}

All patients with confirmed pathological diagnosis of EOC at Dr. Hasan Sadikin General Hospital in the period of 2013 to 2014 were registered. Only patients with complete hematologic data before and after chemotherapy were collected and compared using the paired nonparametric Wilcoxon and McNemar tests.

\section{RESULTS}

In total there were 147 patients with EOC (median age $46 \pm 12$ years), with the most dominant pathological diagnosis of mucinous $(32.7 \%)$ and serous $(29.3 \%)$ types. Only 33 patients had hematologic data before the initiation of chemotherapy. There was a significant decrease after chemotherapy including hemoglobin level (12.0 vs $10.9 \mathrm{~g} / \mathrm{dL}, \mathrm{p}=0.013)$, erythrocyte count (4.53 vs 3.74 million $/ \mu \mathrm{L}, \mathrm{p}<0.001)$, leukocyte count $\left(7,700\right.$ vs $\left.4,000 / \mathrm{mm}^{3} \mathrm{p}<0.001\right)$ and platelet count $(343,000$ vs $215,000 /$ $\left.\mathrm{mm}^{3}, \mathrm{p}<0.001\right)$. Interestingly, anemia cases after chemotherapy were predominant $(87.9 \%)$ compared with erythopenia, leukopenia, thrombocytopenia i.e. $39.4 \%, 57.6 \%$, and $27.3 \%$ respectively.

\section{CONCLUSIONS}

This study confirmed the hematologic toxicities after paclitaxelcarboplatin chemotherapy in EOC patients treated in Hasan Sadikin General Hospital, West Java. The hemoglobin concentration may serve as prognostic factor. Further studies directed to other factors such as genetic factor for polymorphisms may be encouraged to explore the decrease of the hematologic indices.

Keywords: Anemia, erythropenia, leukopenia, thrombocytopenia, epithelial ovarian cancer, paclitaxel-carboplatin
*Center for The Study of Oncology, Faculty of Medicine,

Universitas Padjadjaran

**Department of Pathology,

Faculty of Medicine,

Universitas Padjadjaran/

Dr. Hasan Sadikin General Hospital, Bandung

***Department of Biochemistry and Molecular Biology,

Faculty of Medicine,

Universitas Padjadjaran

****Department of Obstetrics and

Gynecology, Faculty of Medicine,

Universitas Padjadjaran/

Dr. Hasan Sadikin General Hospital,

Bandung

\section{Corresponding:}

Afandi Charles

Center for The Study of Oncology

Faculty of Medicine,

Universitas Padjadjaran

Jl. Raya Bandung Sumedang Km.21,

Jatinangor, West Java, Indonesia.

Phone and fax number:

$+6282297446690$

Email: afandi.charles@gmail.com

Univ Med 2016;35:165-70

DOI: 10.18051/UnivMed.2016.v35.165-170 pISSN: 1907-3062 / eISSN: 2407-2230

This open access article is distributed under a Creative Commons Attribution-Non Commercial-Share Alike 4.0 International License 


\section{INTRODUCTION}

Epithelial ovarian cancers (EOC) are still burdening female health nowadays. The World Health Organization estimated that this type of cancer accounted for 151,905 female deaths worldwide in 2012.(1) In Indonesia, EOC, together with other ovarian cancers are placed in the third rank of the most prevalent gynecologic cancers, next to breast and cervical cancers. ${ }^{(2)}$ There are several risk factors associated with EOC, for example age older than 50 years, no pregnancy and lactation history and family history of breast and ovarian cancers. ${ }^{(3)}$

Management of EOC consists of explorative surgery and chemotherapy. ${ }^{(3)}$ The standard regimen for EOC is intravenous paclitaxel 175 $\mathrm{mg} / \mathrm{m}^{2}$ and carboplatin area under curve is 7.5 for six cycles. ${ }^{(3)}$ These regimen components, especially carboplatin have several side effects in bone marrow, resulting in suppression of hematopoiesis. Common hematologic toxicities developing in patients include anemia, thrombocytopenia, and leukopenia. ${ }^{(4)}$ These side effects are mediated by the formation of DNAprotein cross-linking that further disrupts cellcycle regulation and suppresses cell growth. ${ }^{(5)}$

Chemotherapy is a standardized process performed at Dr. Hasan Sadikin Hospital, Bandung. There is a need for research on the hematologic impact of the paclitaxel- carboplatin combined therapy in EOC patients.

Therefore, the aim of this study was to explore EOC patients treated in Dr. Hasan Sadikin General Hospital, Bandung and to investigate the effect of the paclitaxel-carboplatin regimen.

\section{METHODS}

\section{Research design}

This study employed an analytical retrospective cohort study design conducted using medical records of patients treated in Dr. Hasan Sadikin General Hospital, Bandung. The medical records were collected in the period of January 2013 to December 2014.

\section{Research subjects}

All subjects with a pathological diagnosis of EOC were recruited for this study. Other types of ovarian cancer including malignant granulosa cell tumor and teratoma were excluded. Clinical, laboratory and chemotherapy data including the number of chemotherapy cycles were recorded.

\section{Laboratory analysis}

As for laboratory data, hematologic findings were collected before the initiation of chemotherapy and after chemotherapy of 6 cycles. Anemia was defined at a $\mathrm{Hb}$ concentration of $<12.0 \mathrm{~g} / \mathrm{dL}$, erythropenia at an erythrocyte count of $<3.6 \mathrm{million} / \mu \mathrm{L}$, leukopenia at a leukocyte count of $<4400 / \mathrm{mm}^{3}$ and thrombocytopenia at a platelet count of $<150,000 / \mathrm{mm}^{3}$. Only patients with complete hematologic data and number of chemotherapy cycles were included.

\section{Statistical analysis}

Descriptive statistics for clinical data and histopathological category of EOC were shown as frequencies. McNemar and Wilcoxon tests were used to determine the association of the hematologic status before and after chemotherapy. For the statistical analyses, the SPSS 22.0 software package (SPSS Inc. Chicago. IL) was used.

\section{Ethical clearance}

The ethical clearance required for this study was granted by the Health Research Ethics Committee, Faculty of Medicine, Universitas Padjadjaran - Dr. Hasan Sadikin General Hospital, Bandung (No. 3010/UN6.C.C1/PP/ 2015).

\section{RESULTS}

In total, there were 147 women with a median age of 46 years and an age range of 14 to 81 years, among whom more than half $(59.2 \%)$ were younger than 50 years, i.e. the cut-off point of menopausal age in Indonesia. The most common histopathological types found in these 
patients were the mucinous and serous types, comprising $32.7 \%$ and $29.3 \%$, respectively. Many of the subjects were lost to follow up; some of them refused to undergo chemotherapy management or did not have complete hematologic laboratory data, resulting in only 33 patients with complete laboratory and chemotherapy data who were further analyzed in this study. Of these EOC patients, most (54.5\%) were diagnosed before the age of 50 years and were already in stage III (51.5\%). Only 12 of 33 patients completed the six cycles of chemotherapy, while others underwent only three to five cycles (Table 1). There was no documentation in the medical records regarding the cause of early termination of the chemotherapy.

The hematologic findings were not normally distributed, except for erythrocyte counts. Therefore the hemoglobin, leukocyte and platelet counts were presented as mean and standard deviation, whereas the erythrocyte count was presented as median and range. The hemoglobin level and the erythrocyte, leukocyte and platelet counts decreased significantly after chemotherapy as depicted in Table 2 .

Using categorical laboratory references, the distribution of patients with anemia, erythropenia, leukopenia, and thrombocytopenia also decreased significantly as shown in Table 3. Interestingly, after chemotherapy there was a predominant increase in anemia in 20 of 33 patients $(60.6 \%)$, while erythropenia, leukopenia, and thrombocytopenia increased only in $13 / 33$ (39.4\%), 19/33 (57.6\%), and 9/33 (27.3\%) of patients, respectively (Table 3 ).
Table 1. Characteristics of epithelial ovarian cancer patients with complete hematologic data $(n=33)$

\begin{tabular}{lc}
\hline \multicolumn{1}{c}{ Characteristi } & n (\%) \\
\hline Age group (year s) & $18(54.5)$ \\
$<50$ & $9(27.3)$ \\
$50-59$ & $6(18.2)$ \\
$\geq 60$ & \\
Location d prim ary tumor & $9(27.3)$ \\
Bilater al & $11(33.3)$ \\
Right & $7(21.2)$ \\
Left & $6(18.2)$ \\
Unclear & \\
Histopathological classification & $12(36.4)$ \\
Mucinous & $12(36.4)$ \\
Serous & $4(12.1)$ \\
Endom etrioid & $1(3.0)$ \\
Clear cell & $1(3.0)$ \\
Mix ed & $3(9.1)$ \\
Undifferentiated & \\
Parity & $5(15.2)$ \\
Nulliparous & $12(36.4)$ \\
Primiparous (1) & $13(39.4)$ \\
Multiparous (2-4) & $3(9.1)$ \\
Grand multiparous (>4) & \\
Abortion & $27(81.8)$ \\
0 & $4(12.1)$ \\
1 & $2(6.1)$ \\
2 & $4(12.1)$ \\
FIGO stage & $5(15.2)$ \\
I & $18(54.5)$ \\
II & $3(9.2)$ \\
III & $3(9.1)$ \\
IV & \\
Recurrent & \\
\hline
\end{tabular}

*FIGO=International Federation of Gynecology and Obstetrics

The age at clinical presentation of EOC was analyzed to determine whether it was associated with the decrease in all post-chemotherapy hematologic findings, but the results showed no association (data not shown).

Table 2. Difference of hematologic findings between pre- and post-chemotherapy

\begin{tabular}{|c|c|c|c|c|c|}
\hline \multirow{3}{*}{ Hema to logic find ings } & \multicolumn{4}{|c|}{ Chemo the rap $y$} & \multirow{3}{*}{$\mathbf{p}$} \\
\hline & \multicolumn{2}{|r|}{ Pre } & \multicolumn{2}{|c|}{ Post } & \\
\hline & Median & Range & Medin & Range & \\
\hline Hemogobin, $(g / d \mathbb{L})$ & 12.0 & $(5.5-14.6)$ & 10.9 & $(5.6-13.9)$ & $0.013 *$ \\
\hline Erythrocyte count (million $/ \mu \mathrm{L})$ & 4.53 & $(2.05-6.46)$ & 3.74 & $(1.91-5.19)$ & $<0.001 *$ \\
\hline Leuk ocyte count (/mm $\left.{ }^{5}\right)$ & 7.7 & $(2.10-18.90)^{* * *}$ & 4.0 & $(28-7.8) * *$ & $<0.001 *$ \\
\hline Platelet count $\left(/ \mathrm{mm}^{3}\right)$ & 343 & $(125-771)^{* * *}$ & 215 & $(87-606) * *$ & $<0.001 *$ \\
\hline
\end{tabular}

*p values were statistically significant. Analysis conducted using Wilcoxon test. Significance level $\mathrm{p}=0.05$

**Values were $\mathrm{x} 1.000$ 
Table 3. Chemotherapy induced anemia, erythropenia, leukopenia and thrombocytopenia in adults

\begin{tabular}{|c|c|c|c|}
\hline \multirow{2}{*}{ Hematologic status } & \multicolumn{2}{|c|}{ Chemotherap $y$} & \multirow{2}{*}{$\mathbf{p}$} \\
\hline & Pre (n) & Post (n) & \\
\hline \multicolumn{4}{|l|}{ Hemoglobin } \\
\hline Low (anemia) & 16 & $29 * *$ & $0.001 *$ \\
\hline Normal & 17 & 4 & \\
\hline \multicolumn{4}{|l|}{ Erythrocyte count } \\
\hline Low (erythropenia) & 5 & $13^{* *}$ & $0.039 *$ \\
\hline Normal & 28 & 20 & \\
\hline \multicolumn{4}{|l|}{ Leuk ocyte count } \\
\hline Low (1euk operia) & 1 & $19 * *$ & $<0.001 *$ \\
\hline Normal & 32 & 14 & \\
\hline \multicolumn{4}{|l|}{ P1atelet count } \\
\hline Low (thrombocytopenia) & 1 & $9 * *$ & $<0.001 *$ \\
\hline Normal & 32 & 24 & \\
\hline
\end{tabular}

${ }^{*} \mathrm{p}$ values were statistically different. Analysis conducted using McNemar test. Significance level $\mathrm{p}=0.05$

**Low hematologic counts after chemotherapy

\section{DISCUSSION}

Our study explored the hematologic findings before and after paclitaxel-carboplatin chemotherapy among patients with epithelial ovarian cancer (EOC) and confirmed that there was a significant decrease in hematologic findings such as anemia, erythropenia, leukopenia, and thrombocytopenia. These findings are coherent with studies in advanced EOC cases, either using conventional or dose-dense paclitaxel-carboplatin regimen. ${ }^{(4,7)}$ The paclitaxel-carboplatin regimen is known to have a lower incidence of high-grade neutropenia in EOC chemotherapy compared to its alternative regimen, docetaxel and carboplatin. ${ }^{(8)}$ In our study we did not perform neutrophil counts; however, the hemoglobin level was significantly reduced after chemotherapy. Hemoglobin levels before chemotherapy have proposed to have prognostic value in terms of progression-free survival time for EOC patients. ${ }^{(9)}$ The paclitaxel-carboplatin regimen is also used in other neoplasm cases, notably non-small cell lung cancer (NSCLC) and urothelial cancer, and has been associated with febrile neutropenia. ${ }^{(10)}$ Furthermore, anemia, neutropenia, and thrombocytopenia are the most frequently observed toxicities after paclitaxel-carboplatin chemotherapy in advanced urothelial cancer patients in Japan. ${ }^{(11)}$ These hematologic toxicities are also observed in NSCLC, and in addition, the paclitaxel-carboplatin regimen has been revealed to produce more severe anemia and neutropenia in Asian than in non-Asian patients. ${ }^{(12)}$

As hematopoietic stem cells undergo senescence, many lesions and mutations are accumulated in their DNA, producing exhaustion in stem cell division. ${ }^{(7)}$ This underlying mechanism may be responsible for the more severe hematologic toxicities of cytotoxic chemotherapy regimens, for example cyclophosphamidedoxorubicin in breast cancer and bevacizumabpaclitaxel-carboplatin in NSCLC. ${ }^{(13,14)}$ However, an association between age and severity was not documented in these studies, and another study in NSCLC cases with paclitaxel-carboplatin chemotherapy also yielded similar results. ${ }^{(15)}$

The hematologic toxicities occurring in EOC patients after having received paclitaxelcarboplatin chemotherapy are influenced by several factors, i.e. from the regimen itself and patient background. Paclitaxel exerts its cytotoxic effects by depolymerizing microtubules, thus arresting the cell cycle and inducing apoptosis. ${ }^{(16)}$ However, paclitaxel only induces minimal hematologic toxicities when administered alone. ${ }^{(16)}$ Carboplatin used in this regimen has a myelosuppressive effect via the mechanism of DNA-protein damage in bone marrow by means of crosslinking between them, thus resulting in decreased numbers of erythrocytes, leukocytes, and platelets, as well 
as other toxic effects (neurotoxicity, ototoxicity, and nephrotoxicity). ${ }^{(17,18)}$ It also seems that carboplatin may increase serum TNF- $\alpha$, suppressing the development of hematologic progenitor cells. ${ }^{(19)}$

Currently, genetic polymorphisms are being investigated to determine their association with paclitaxel-carboplatin hematologic toxicity, depending on race and ethnicity. The single nucleotide polymorphisms $2677 \mathrm{G}>\mathrm{T} / \mathrm{A}$ and $3435 \mathrm{C}>\mathrm{T}$ of $A B C B 1$ genes have been associated with neutropenia in paclitaxel chemotherapy in EOC cases in some studies, ${ }^{(20,21)}$ but no association has been found in others. ${ }^{(22,23)}$ Some studies in NSCLC have demonstrated that polymorphisms in drug-transporter genes ( $A B C B 1$ and $A B C G 2$ ), DNA-repair pathway genes (ERCC4 and $X C C$ ), and apoptosis pathway genes (CASP 8 and $C A S P 10)$ are associated with thrombocytopenia, neutropenia, and severe hematologic toxicity, respectively, due to paclitaxel-carboplatin effects. ${ }^{(24-26)}$

One limitation of this study is the small sample size which was not appropriate to the estimated sample size due to incomplete data. Another limitation is inadequate hematologic data in each of the chemotherapy cycles, which limits statistical analysis; however, the decreasing trend in the hematologic data might be used to generalize the findings.

\section{CONCLUSION}

In conclusion, the decrease in hematologic data such as anemia, leukopenia, and thrombocytopenia after paclitaxel-carboplatin chemotherapy were confirmed in EOC patients treated in Dr. Hasan Sadikin General Hospital. The hemoglobin concentration may serve as prognostic factor. In addition, further studies are needed on genetic background factors that may play a role in the decrease in hematologic data.

\section{CONFLICT OF INTEREST}

Competing interest: No relevant disclosures.

\section{ACKNOWLEDGEMENT}

This research is part of a Progress Grants 2014 study conducted under the Oncology Working Group, Faculty of Medicine, Universitas Padjadjaran.

\section{REFERENCES}

1. Union for International Cancer Control. Epithelial ovarian cancer. Geneva: World Health Organization;2014.

2. Wahidin M, Noviani R, Hermawan S, et al. Population-based cancer registration in Indonesia. Asian Pac J Cancer Prev 2012;13: 1709-10.

3. Jelovac D, Armstrong DK. Recent progress in the diagnosis and treatment of ovarian cancer. CA 2011;61:183-203.

4. Katsumata N, Yasuda M, Takahashi F, et al. Dose-dense paclitaxel once a week in combination with carboplatin every 3 weeks for advanced ovarian cancer: a phase 3 , openlabel, randomised controlled trial. Lancet 2009;374:1331-8.

5. Barker S, Weinfeld M, Murray D. DNAprotein crosslinks: their induction, repair, and biological consequences. Mutat Res 2005;589:111-35.

6. Siregar MFG. Perimenopausal and postmenopausal complaints in paramedics assesed by menopause rating scale in Indonesia. J Dental Med Sci 2009;13:38:42.

7. Van der Burg M, Vergote I, Onstenk W, et al. Long-term results of weekly paclitaxel carboplatin induction therapy: an effective and well-tolerated treatment in patients with platinum-resistant ovarian cancer. Eur J Cancer 2013;49:1254-63.

8. Pignata S, Cannella L, Leopardo D, et al. Chemotherapy in epithelial ovarian cancer. Cancer Lett 2011;303:73-83.

9. Eichbaum MH, Weiss LM, Bruckner T, et al. Prognostic impact of hemoglobin levels before and during carboplatin/taxane-based chemotherapy in patients with primary invasive epithelial ovarian cancer. Med Sci Monit Basic Res 2009;15:CR156-CR63.

10. Aapro MS, Cameron DA, Pettengell R, et al. EORTC guidelines for the use of granulocyte-colony stimulating factor to reduce the incidence of chemotherapyinduced febrile neutropenia in adult patients 
with lymphomas and solid tumours. Eur J Cancer 2006;42:2433-53.

11. Soga N, Onishi T, Arima K, et al. Paclitaxel carboplatin chemotherapy as a second line chemotherapy for advanced platinum resistant urothelial cancer in Japanese cases. Int J Urol 2007;14:828-32.

12. Hasegawa Y, Kawaguchi T, Kubo A, et al. Ethnic difference in hematological toxicity in patients with non-small cell lung cancer treated with chemotherapy: a pooled analysis on Asian versus non-Asian in phase II and III clinical trials. J Thorac Oncol 2011;6: 1881-8.

13. Ramalingam SS, Dahlberg SE, Langer CJ, et al. Outcomes for elderly, advanced-stage non-small-cell lung cancer patients treated with bevacizumab in combination with carboplatin and paclitaxel: analysis of Eastern Cooperative Oncology Group Trial 4599. J Clin Oncol 2008;26:60-5.

14. Muss HB, Berry DA, Cirrincione C, et al. Toxicity of older and younger patients treated with adjuvant chemotherapy for nodepositive breast cancer: the Cancer and Leukemia Group B Experience. J Clin Oncol 2007;25:3699-704.

15. Ganti AK, Loberiza FR Jr, Kessinger A. Factors affecting bone marrow toxicity following administration of carboplatin and paclitaxel in patients with non-small cell lung cancer. Anticancer Res 2010;30:1365-9.

16. Kampan NC, Madondo MT, McNally OM, et al. Paclitaxel and its evolving role in the management of ovarian cancer. BioMed Res Int 2015;2015:413076.

17. Hildebrandt MA, Gu J, Wu X. Pharmacogenomics of platinum-based chemotherapy in NSCLC. Expert Opin Drug Metab Toxicol 2009;5:745-55.

18. Joerger M, Huitema AD, Richel DJ, et al. Population pharmacokinetics and pharmacodynamics of paclitaxel and carboplatin in ovarian cancer patients: a study by the European organization for research and treatment of cancer-pharmacology and molecular mechanisms group and new drug development group. Clin Cancer Res 2007; 13:6410-8.

19. Arafa HM, Ismail RS, Nabil N, et al. Carnitine deficiency: a causative clue or a sequel in carboplatin myelosuppression. J Cancer Res Updates 2014;3:226-35.

20. Sissung TM, Mross K, Steinberg SM, et al. Association of ABCB1 genotypes with paclitaxel-mediated peripheral neuropathy and neutropenia. Eur J Cancer 2006;42:28936.

21. Bergmann TK, Brasch Andersen C, Gréen $\mathrm{H}$, et al. Impact of $\mathrm{ABCB} 1$ variants on neutrophil depression: a pharmacogenomic study of paclitaxel in 92 women with ovarian cancer. Basic Clin Pharmacol Toxicol 2012; 110:199-204.

22. Bergmann TK, Gréen H, Brasch-Andersen $\mathrm{C}$, et al. Retrospective study of the impact of pharmacogenetic variants on paclitaxel toxicity and survival in patients with ovarian cancer. Eur J Clin Pharmacol 2011;67:693700.

23. Marsh S, Paul J, King CR, et al. Pharmacogenetic assessment of toxicity and outcome after platinum plus taxane chemotherapy in ovarian cancer: the Scottish randomised trial in ovarian cancer. J Clin Oncol 2007;25:4528-35.

24. Qian J, Qu H-Q, Yang L, et al. Association between CASP8 and CASP10 polymorphisms and toxicity outcomes with platinum-based chemotherapy in Chinese patients with non-small cell lung cancer. Oncologist 2012;17:1551-61.

25. Lamba JK, Fridley BL, Ghosh TM, et al. Genetic variation in platinating agent and taxane pathway genes as predictors of outcome and toxicity in advanced non-smallcell lung cancer. Pharmacogenomics 2014;15:1565-74.

26. Gu S, Wu Q, Zhao X, et al. Association of CASP3 polymorphism with hematologic toxicity in patients with advanced non small cell lung carcinoma treated with platinum based chemotherapy. Cancer Sci 2012;103: 1451-9. 